\title{
Media literacy, coding e cittadinanza digitale: apprendere e costruire con le tecnologie
}

\section{Alfabetização midiática, codificação e cidadania digital: aprender e construir com as tecnologias}

Mario Pireddu*

\section{Sommario}

Il complesso rapporto tra educazione e tecnologia digitale può essere compreso se è chiaro il ruolo delle tecnologie nell'ecosistema di rete che abitiamo. Le tecnologie non sono strumenti o aiuti esterni al corpo umano, ma agenti di trasformazione delle nostre strutture mentali e corporee. II concetto di fluidità computazionale aiuta a superare i limiti delle teorie relative al pensiero computazionale: il coding può essere visto a tutti gli effetti come una forma di espressione e di padronanza di un linguaggio, secondo un approccio incentrato sulla progettazione, il pensiero critico e la creatività. Lo scopo delle attività di coding non è imparare abilità e concetti base dell'informatica, ma l'espressione di se stessi attraverso ambienti di sviluppo creativo. Padroneggiare il coding aiuta a sviluppare il proprio pensiero, a sviluppare la propria espressività e a sviluppare la propria identità. La fluidità computazionale ha a che fare non solo con la comprensione dei concetti computazionali e delle strategie di risoluzione dei problemi, ma anche con la capacità di saper creare e sapere come esprimersi con le tecnologie digitali per contribuire attivamente alla società verso una piena cittadinanza digitale.

Parole chiave: Educazione. Tecnologia. Coding. Costruzionismo. Pensiero.

\section{Resumo}

A complexa relação entre educação e tecnologia digital pode ser entendida se o papel das tecnologias no ecossistema da rede que habitamos for claro. As tecnologias não são ferramentas ou auxílios externos ao corpo humano, mas agentes de transformação de nossas estruturas mentais e corporais. O conceito de fluidez computacional ajuda a superar as limitações das teorias relacionadas ao pensamento computacional: a codificação pode ser vista em todos os aspectos como uma forma de expressão e domínio de uma linguagem, de acordo com uma abordagem focada em design, pensamento crítico e criatividade. O objetivo da codificação de atividades não é aprender habilidades e conceitos básicos de computação, mas, sim, expressar-se por intermédio de ambientes de desenvolvimento criativo. Dominar a codificação ajuda a desenvolver o próprio pensamento, a própria expressividade e a própria identidade. A fluidez computacional tem a ver não só com a compreensão de conceitos computacionais e estratégias de resolução de problemas, mas também com a capacidade de criar e saber se expressar com tecnologias digitais, para contribuir ativamente para a sociedade em direção à plena cidadania digital.

Palavras-chave: Educação. Tecnologia. Codificação. Construcionismo. Pensamento.

Recebido em 30/09/2018 - Aprovado em 27/02/2019

http://dx.doi.org/10.5335/rep.v26i2.8704

Ricercatore del Dipartimento di Scienze dell'educazione, Università di Roma tre, Italia. Posta elettronica: mario.pireddu@unitus.it 
Per riflettere sul complesso rapporto tra educazione e tecnologia digitale può essere utile partire da una considerazione relativa ai processi di alfabetizzazione. Per migliaia di anni la maggior parte delle persone sul pianeta non ha potuto sviluppare competenze alfabetiche e scrittorie, restando esclusa dalle specifiche pratiche di addestramento previste per l'interiorizzazione delle stesse logiche alfabetiche. Oggi a questo proposito, dopo decenni di politiche pubbliche e di industria culturale, la ricerca in campo neurocognitivo sottolinea il ruolo della plasticità sinaptica del cervello, la capacità di adattarsi agli strumenti che di volta in volta utilizziamo. Se per la vista e il linguaggio sappiamo che esistono geni specifici, così non è per la lettura: diventiamo abili lettori e scrittori grazie alla capacità delle sinapsi di modificare la struttura e la funzionalità del sistema nervoso in base all'esperienza (WOLF, 2012, 2018).

Walter J. Ong (1986), nel suo celebre studio sul rapporto tra oralità e scrittura, scrisse che la scrittura fu l'evento di maggiore importanza nella storia delle invenzioni tecnologiche dell'uomo. Tutt'altro che una semplice appendice del discorso orale, la scrittura ha consentito l'apertura verso una nuova dimensione del sensorio, trasformando allo stesso tempo discorso e pensiero attraverso la vista (ONG, 1986, p. 126-127). In quel testo lo studioso gesuita ricordava come la tecnologia della scrittura abbia richiesto nel tempo l'uso di una serie di strumenti quali penne, pennelli, superfici predisposte, tavolette, pelli, inchiostro colori e diverse altre cose. Ong opponeva la scrittura al linguaggio "naturale" dell'oralità, ricordando che non vi è modo di scrivere naturalmente.

In quest'ottica tutte le tecnologie possono essere viste come intrinsecamente artificiali, ma - paradossalmente - la dimensione dell'artificialità emergerebbe come naturale per gli esseri umani. Tecnologie non come aiuti esterni, dunque, ma agenti di trasformazione delle nostre strutture mentali e corporee (MORIGGI, 2014; MARAGLIANO; PIREDDU, 2012). È questa la lezione dell'antropologia filosofica, della cibernetica, delle riflessioni novecentesche sui media, da Walter Benjamin a Marshall McLuhan, e delle elaborazioni pedagogiche di Seymour Papert e del costruzionismo. A questo proposito è bene sgombrare il campo dal "nuovismo" di cui è intriso il dibattito attuale sulle tecnologie digitali per l'educazione. Secondo McLuhan una tecnologia può essere definita "nuova" unicamente quando rimette in discussione le gerarchie tra i sensi, ora privilegiandone alcuni, ora "narcotizzandone" altri (cfr. MCLUHAN, 1997). Sappiamo che non si possono considerare i singoli sensi - vista, udito, tatto, olfatto, gusto - come perfettamente isolati l'uno dall'altro: ciò che accade nell'organismo alla comparsa di un medium 
nuovo, dunque, sarebbe una redistribuzione del peso che alcune zone del corpo hanno nell'insieme di quello che in altri ambiti di riflessione viene definito "schema corporeo" di un individuo. Qui il riferimento è agli studi di fenomenologia della percezione di Maurice Merleau-Ponty (1965, p. 174): lo studioso francese parlava di "esperienza integrale" del soggetto, ricordando che non possono esistere un'esperienza tattile o un'esperienza visiva scisse dall'insieme delle dimensioni percettive. Ogni tecnologia, ogni medium entra in diretto contatto con il corpo, con la stessa esperienza del corpo nel mondo (il fenomenologico être au mond). Se per il filosofo francese è il corpo a "comprendere", per McLuhan, come è noto, le tecnologie e i media sono estensioni dell'organismo umano, "risorse naturali", protesi. Per Seymour Papert, allievo di Jean Piaget che ha contribuito alla elaborazione della teoria costruzionista dell'apprendimento, i computer - software e tecnologie digitali - consentono di incrementare nei bambini e negli adulti il potere di sperimentare, esplorare ed esprimersi. Le persone sono in grado di costruire conoscenze in modo più efficace se attivamente coinvolte nel costruire veri e propri artefatti cognitivi, $\mathrm{e}$ le tecnologie digitali possono essere d'aiuto nel pensare al proprio modo di pensare, dunque nell'imparare di più sul proprio modo di imparare.

Tornando quindi alla scrittura dal punto di vista della ricerca in campo neurocognitivo, negli ultimi anni gli studi hanno mostrato ancora una volta l'adattabilità del cervello umano nel rispondere al nuovo ecosistema comunicativo digitale. Le connessioni neuronali costruite per la lettura su carta vengono alterate per svilupparne altre più adatte a fronteggiare la continua e spesso vorticosa offerta di contenuti in ambienti digitali. Secondo Maryanne Wolf (2018), che studia da anni le connessioni tra cervello e informazione digitale, l'adattabilità del cervello ci consente di gestire oggi un accesso quasi universale al sapere e una più efficace gestione delle relazioni sociali, con tuttavia il rischio di una contemporanea perdita di qualità umane fondamentali come il pensiero critico, l'introspezione, l'empatia e l'immaginazione creativa.

Se da un lato va riconosciuto che queste ultime qualità non sembrano essere state al centro delle società che hanno preceduto quella attuale, dall'altro è sicuramente vero che in ogni epoca chi si è occupato e si occupa di educazione e formazione ha lavorato e deve lavorare per il loro potenziamento. È quanto cerca di fare, sulla scia del lavoro iniziato da Papert, il gruppo di lavoro del Lifelong Kindergarten presso il Media Lab del MIT diretto da Mitchel Resnick (2018). Il riferimento alla scrittura e alle implicazioni dell'alfabetizzazione viene utilizzato dallo stesso Resnick per spiegare il senso delle attività legate alla programmazione 
e alla creatività attraverso il software Scratch, erede del Logo creato da Papert negli anni Sessanta del secolo scorso. Opponendo il concetto di fluidità computazionale a quello più abusato di pensiero computazionale, Resnick immagina il coding come una forma di espressione e di padronanza di un linguaggio. Come nel caso della scrittura - per cui quando si impara a scrivere non è sufficiente imparare grammatica, punteggiatura e ortografia -, quando si lavora sul coding non ci si può limitare ai puzzle o all'apprendimento del funzionamento di base del linguaggio di programmazione. Se per saper concretamente scrivere bene è imprescindibile imparare a raccontare storie e a comunicare le proprie idee, per la fluidità computazionale è necessario un approccio incentrato sulla progettazione. Incoraggiati a creare storie interattive, giochi e animazioni di vario tipo, i ragazzi trasformano le proprie idee in progetti che possono condividere con altre persone. Non si è davanti alla mera creazione di un programma allo scopo di raggiungere obiettivi prefissati, con cui imparare abilità e concetti base dell'informatica. Si è invece più vicini all'espressione di sé stessi attraverso ambienti di sviluppo creativo: secondo il gruppo di ricerca di Resnick padroneggiare la scrittura e il coding aiuta a sviluppare il proprio pensiero, a sviluppare la propria espressività e a sviluppare la propria identità. Proprio come risolvere le parole crociate non porta a saper raccontare storie e ad esprimere le proprie idee con padronanza, la mera competenza tecnica delle basi del coding non garantisce lo sviluppo di pensiero critico e creativo. Da questo punto di vista, la fluidità computazionale ha a che fare non solo con la comprensione dei concetti computazionali e delle strategie di risoluzione dei problemi, ma anche con la capacità di saper creare e sapere come esprimersi con le tecnologie digitali. Se con i processi di scrittura si impara a organizzare, raffinare, e riflettere sulle proprie idee - e dunque si diventa migliori pensatori -, quando si impara a programmare si impara a individuare problemi e risolverli, si impara come suddividere e scomporre problemi complessi in parti più semplici, e si diventa capaci di migliorare i propri progetti nel tempo. In buona sostanza, anche quando si impara a programmare si può diventare migliori pensatori.

Resnick sviluppa il paragone con l'alfabetizzazione fino a sostenere che il coding sia una vera e propria estensione della scrittura, in grado di consentire di scrivere storie interattive, animazioni, giochi e simulazioni.

É chiaro come anche la tecnologia digitale, al pari di quelle che la hanno preceduta, continui a consentire l'apertura verso nuove dimensioni del sensorio, trasformando allo stesso tempo discorso e pensiero attraverso il coinvolgimento 
attivo di tutto il corpo. I sistemi educativi non si sono ancora aperti alle opportunità offerte da percorsi di apprendimento costruzionisti, e la versione povera del coding a cui fa riferimento Resnick è quella che si è diffusa maggiormente tra le pratiche scolastiche. Seymour Papert (1994) agli inizi degli anni Novanta del secolo scorso lamentava la "tendenza connaturata" della scuola a rendere infantili i bambini, messi in condizione di dover seguire le condizioni altrui e di svolgere compiti dettati da altri spesso privi di alcun valore intrinseco. Per il matematico e pedagogista sudafricano le criticità dell'introduzione dei computer nelle scuole furono evidenti sin dall'inizio: aver relegato i computer in sale dedicate all'informatica da utilizzare in orari definiti ha comportato la trasformazione di pratiche interattive e di scoperta in materia o disciplina tra le altre, regolata da specifici programmi ministeriali. La conseguenza - se non l'obiettivo - di tale integrazione è stata la neutralizzazione della forza destabilizzante delle tecnologie digitali per la formazione. Le critiche mosse da Papert non riguardavano le aule di informatica e il loro utilizzo, ma il fatto che quelle stanze isolate non venivano usate come punto di incontro di idee che prima venivano tenute separate.

Il pensiero di Papert prende corpo nell'intersezione tra tre aree disciplinari: quella matematica, quella informatica e quella pedagogica. Quest'ultima in modo particolare è determinante, perché permette da un lato di costruire percorsi di riflessione e interventi pratici eterogenei e dall'altro di lavorare sul tema dell'apprendimento da un punto di vista inedito.

L'esperienza di Papert a Ginevra con Jean Piaget, tra la fine degli anni Cinquanta e i primi anni Sessanta del secolo scorso, è essenziale per la costruzione di un pensiero e di una proposta teorica autonomi. Per Piaget era essenziale considerare gli individui, in particolare durante l'infanzia, portatori di un bagaglio di conoscenze ed elementi noti, in grado di essere attivati e di modificarsi nel tempo. La capacità cognitiva quindi non ha a che fare con la semplice trasmissione di informazioni o con la replicazione di comportamenti altrui. Nell'apprendimento sono in gioco dunque costanti processi di appropriazione, confronto con la propria esperienza ed eventuali integrazioni nel proprio schema mentale di nuovi elementi e nuovi comportamenti. L'atto di apprendere ha a che fare dunque con processi cognitivi e corporei, nei bambini come negli adulti, per i quali non si abbandona una propria teoria in base alla semplice trasmissione di una teoria migliore e più efficace. L'evoluzione delle capacità cognitive per Piaget avviene mediante continue azioni di confronto e analisi con il proprio sapere e con le proprie esperienze. Dal punto di vista didattico, ciò significa consentire a chi apprende di poter sperimentare, 
di essere soggetto attivo e partecipe delle diverse esperienze di apprendimento. Per farlo occorre ripensare sia l'azione didattica che il contesto entro il quale tale azione viene svolta: la dimensione laboratoriale offre la possibilità di poter dare corpo a pratiche finalizzate alla costruzione di una conoscenza fondata sulla sperimentazione, sul cimentarsi attivamente con la comprensione di un concetto, di un evento o di un problema. Conoscere non può dunque coincidere con la mera trasmissione di informazioni: si tratta di una dimensione che si costruisce invece potendo compiere esperienza mediante l'interazione concreta con il mondo, le persone e gli oggetti. La posizione costruttivista di Piaget tiene conto anche delle possibili resistenze all'apprendimento, solitamente messe in atto da chi dovrebbe apprendere, e della necessità per chi si occupa di didattica di prendere in considerazione tali resistenze per non sacrificare i propri obiettivi didattici. Papert muove da queste premesse e porta avanti il proprio percorso di ricerca verso un'idea di costruzionismo capace di tenere insieme i differenti ambiti disciplinari di cui si è detto in precedenza (matematica, informatica, pedagogia). Da qui lo sviluppo e la definizione di una struttura teorica dedicata all'apprendimento, e l'utilizzo delle macchine come strumenti dedicati alla costruzione di percorsi di apprendimento efficaci e in grado di favorire la riflessione cognitiva e metacognitiva sulle esperienze in corso, la comprensione dei perché e dei come delle proprie azioni. Nel riflettere sulle situazioni e sui problemi, gli studenti integrano le nuove esperienze e le nuove informazioni con la loro precedente conoscenza del mondo, nel costante lavoro per dare un senso a ciò che osservano. Costruiscono i propri modelli mentali attraverso cui spiegano ciò che analizzano, e li rendono sempre più complessi con l'esperienza e l'ulteriore riflessione dando vita a rappresentazioni mentali più articolate.

L'interesse è qui per le modalità attraverso le quali prende forma e si trasforma il sapere in contesti specifici, per i mutamenti nei processi mentali che vengono messi in gioco per imparare e come questi possano variare attraverso l'interazione con differenti media. Nelle attività didattiche ideate da Papert l'oggetto o il dispositivo tecnologico come facilitatori dell'apprendimento sono infatti elementi centrali. L'analisi, la manipolazione, la costruzione di oggetti rappresentano processi cognitivi fondamentali per la maturazione di un apprendimento. Dietro l'idea di artefatto cognitivo vi è il riferimento alla mente che per apprendere - a qualunque età - ha bisogno di costruire oggetti e dispositivi, e dunque di maneggiare materiali reali. Nell'ottica costruzionista diventa centrale il procedere per prove ed errori, mediante serie di tentativi di rappresentazione del mondo che ci circonda. L'apprendimento deve quindi avere a che fare con il confronto, l'analisi, la discussione, l'esposizione, 
la costruzione, lo smontaggio e la ricostruzione degli artefatti cognitivi e con la metacognizione. Costruire un oggetto e interagire con un dispositivo diventano processi utili per sviluppare idee e per imparare: si affinano abilità, si definiscono percorsi mentali per la risoluzione di un problema o per il raggiungimento di un obiettivo. Costruire, smontare, utilizzare, manipolare e analizzare oggetti comporta anche, come si è detto, l'esposizione, il confronto e la discussione con gli altri: qui la relazione assume una valore centrale nel processo di apprendimento. Attraverso gli artefatti cogniti prende corpo una matrice relazionale sulla quale costruire saperi e competenze: interazione, scambio e analisi degli errori riscontrati; formulazione di ipotesi da testare e mettere in pratica; individuazione di errori e condivisione delle osservazioni e delle riflessioni. Anche se qualsiasi oggetto, dispositivo o materiale può assumere il ruolo di artefatto cognitivo se opportunamente collocato in una dimensione di apprendimento, è vero che Papert si è concentrato tra i primi sulle opportunità offerte dalle tecnologie digitali per la costruzione di percorsi didattici efficaci e innovativi. Le sue riflessioni sul rapporto tra computer e scuola sono per certi versi ancora attuali: l'ostilità verso la concezione dell'informatica come "disciplina" tra le altre e al confinamento del computer in appositi laboratori da utilizzare soltanto in orari specifici mostrava la convinzione della necessità di adottare i computer e i linguaggi digitali in modo trasversale. Solo in questo modo per Papert la scuola avrebbe potuto sfruttare appieno le opportunità offerte dalla flessibilità delle tecnologie digitali, senza neutralizzarne la portata: l'obiettivo era ripensare interamente la visione meramente tecnica delle macchine, verso una didattica digitalmente aumentata in grado di lavorare sulla scoperta e sulla creatività.

Oggi, più di venticinque anni dopo i rilievi di Papert, in molti paesi i sistemi educativi sono reduci dall'attraversamento di diverse riforme, con alcuni passaggi chiave come il superamento dei vecchi programmi ministeriali e l'elaborazione di percorsi costruiti per lavorare sull'integrazione di competenze e conoscenze. Sul tema specifico della digitalizzazione della scuola e dei sistemi educativi sono stati fatti alcuni passi avanti ma è mutato radicalmente anche l'ecosistema comunicativo di riferimento e le stesse infrastrutture della conoscenza non sono più le stesse. Oggi il sapere risiede sempre meno all'interno delle tradizionali strutture che per secoli sono state incaricate della sua produzione e conservazione, e sempre più abita le reti sotto forma di dati, metadati, big data e relazioni (RONCAGLIA, 2018; FLORIDI, 2017; PIREDDU, 2017b; WEINBERGER, 2012; MANOVICH, 2010). Neanche tanto paradossalmente il discorso di Papert, ripreso dal gruppo 
di ricerca del Media Lab al MIT, si mostra quindi ancora più attuale. Sembrano riconoscerlo i gruppi di lavoro della Commissione Europea, che negli ultimi anni hanno prodotto e pubblicato due framework dedicati alle competenze digitali necessarie da un lato ai cittadini e dall'altro agli educatori del XXI secolo. Si tratta del framework europeo DigComp (CARRETERO GOMEZ; VUORIKARI; PUNIE, 2017), aggiornato periodicamente e dedicato all'individuazione delle competenze chiave da promuovere, consolidare, validare e certificare per i cittadini, e del framework DigCompEdu (JOINT RESEARCH CENTRE, 2017), proposto come riferimento europeo per l'implementazione di linee guida nazionali dei paesi membri e per la progettazione di percorsi formativi all'altezza dei mutamenti sociali, antropologici, economici e culturali degli ultimi anni. Il DigCompEdu nasce dalla presa d'atto dell'ubiquità e della pervasività dei dispositivi digitali, e del relativo dovere per gli educatori di essere competenti in ambito digitale per poter aiutare gli studenti a diventarlo a loro volta.

Le competenze elencate nel framework DigCompEdu sono più di venti, raggruppate in sei aree: Professional engagement, Digital Resources, Digital Pedagogy, Digital Assessment, Empowering Learners, Facilitating Learners' Digital Competence. Tra le competenze indicate vi è il saper riflettere sull'uso delle tecnologie nelle proprie pratiche didattiche; sapere come ricercare, selezionare e valutare risorse digitali più utili per la didattica; saper organizzare, condividere e pubblicare risorse digitali aperte; saper creare e editare contenuti digitali pensati per la didattica; saper progettare e supportare attività collaborative tra gli studenti; gestire la propria auto-formazione, conoscere le metodologie della ricerca didattica per la raccolta e l'analisi di dati utili a valutare l'efficacia dei percorsi proposti; saper utilizzare gli ambienti per gestire i feedback per gli studenti, al fine di ricalibrare e individualizzare l'insegnamento; saper gestire le problematiche relative all'accessibilità e saper utilizzare spazi, ambienti e risorse più utili allo scopo. Naturalmente uno degli obiettivi cardine, come si è detto, è promuovere lo sviluppo della media literacy, della competenza digitale degli studenti: aiutare a sviluppare capacità di comunicazione e collaborazione anche attraverso ambienti digitali e di rete, favorire la creazione di contenuti e la risoluzione di problemi di vario tipo. In Italia alcuni dei questi punti chiave sono stati recepiti con il Piano Nazionale Scuola Digitale contenuto nella legge 107/2015 (PIREDDU, 2017a), e con il sillabo di Generazioni Connesse dedicato all'educazione civica digitale.

Come sottolinea Sonia Livingstone (2016), studiosa della London School of Economics a capo di diversi progetti di ricerca mondiali ed europei dedicati al 
rapporto tra bambini, ragazzi e dimensioni della socialità online, è imprescindibile comprendere i tipi di interazione sociale che i ragazzi prediligono e desiderano, così le modalità attraverso cui i media digitali consentono loro di gestire queste interazioni e le distanze tra percezione e realtà. Se, al di là delle sterili opposizioni che a volte ancora caratterizzano il dibattito su questi temi, le relazioni e le conversazioni face-to-face dei più giovani vengono riconfigurate e non sostituite attraverso gli ambienti digitali, ecco allora che per chi si occupa della loro formazione è necessaria una comprensione profonda di tali ambienti e delle loro logiche di fondo (GEE JAMES, 2013; LANKSHEAR; KNOBEL, 2008). In questo senso la proposta del Lifelong Kindergarten per un approccio critico e creativo al digitale appare ancora una volta come la più accorta: dall'imparare a programmare (learn to code) al programmare per imparare (code to learn). Questa seconda impostazione oltrepassa la strumentalità della prima, e con essa delle versioni povere del coding che predomina anche nelle scuole italiane. Seguendo l'impostazione data da Papert, ci si allontana dai modelli in cui è il computer a programmare i bambini, per abbracciare prassi concrete in cui è il bambino a programmare il computer. L'obiettivo è far acquisire ai bambini le competenze necessarie per utilizzare le tecnologie digitali, e stabilire allo stesso tempo una reale comprensione di concetti profondi legati alla scienza, alla matematica e all'arte della costruzione di modelli intellettuali. Tra le conclusioni più interessanti del costruzionismo di Papert e allievi, si è detto, vi è la ridefinizione del processo di apprendimento: attraverso la programmazione i bambini possono giungere spontaneamente a scoprire - o a riscoprire - autonomamente e a fare proprie diverse leggi matematiche complesse. Possono riuscire a interiorizzare idee chiave come la scomposizione di problemi grandi in parti più piccole, o ancora trovare soluzioni tramite tentativi, errori e debugging (individuazione e correzione di errori). I principi pedagogici del costruzionismo sommano a quelli del più classico costruttivismo l'elaborazione di artefatti cognitivi e la produzione di oggetti tangibili che trovano concretizzazione nelle pratiche di costruzione di oggetti, personaggi, mondi e storie da parte dei bambini.

L'apprendimento è costruito come un processo di esplorazione, creazione e costruzione, e lo sviluppo di conoscenza prende corpo attraverso l'interazione con il mondo circostante e con le altre persone. L'apprendimento è dunque realtà resa possibile dal confronto, dalla discussione, dall'esposizione, dall'osservazione, dall'analisi, dalla costruzione, dallo smontaggio e dalla ricostruzione degli artefatti cognitivi. Papert parlava esplicitamente della necessità di concentrarsi 
sull'apprendimento più che sull'insegnamento (teaching vs learning), perché insegnare è importante ma apprendere lo è molto di più. Il portato concettuale è una visione dell'apprendimento come costruzione e ricostruzione continua più che come trasmissione di conoscenze. Un apprendimento che, al di là di ogni idealizzazione o eccessiva astrazione, è inevitabilmente un processo "sporco" (dirty learning) attraverso cui costruire saperi e conoscenze condivise. Da matematico, Papert si accorse che uno dei problemi più grandi per l'apprendimento della matematica a scuola era la differenza con quella che definiva "la matematica del mondo reale". Nel mondo reale, la matematica viene regolarmente utilizzata per la costruzione di ponti o macchine di vario tipo, per produrre teorie e spiegazioni sul funzionamento degli atomi e sull'origine degli astri e dell'universo, o anche solo per la gestione dell'economia domestica o aziendale. A scuola, invece, i bambini non sempre imparano a fare molte cose con la matematica, presi in attività che non li coinvolgono più di tanto. Tra gli obiettivi principali del lavoro di Papert e del suo gruppo di lavoro, e oggi del Lifelong Kindergarten del MIT, vi è quello di cercare modi per rendere possibile per i bambini fare qualcosa di interessante e sentire il proprio rapporto con la matematica più simile a quello degli scienziati, degli ingegneri e di tutte le persone che "utilizzano la matematica costruttivamente per costruire qualcosa".

Allo stesso modo, immergersi oggi in ambito scolastico negli ambienti digitali e in modo attivo è utile per la formazione di cittadini pienamente consapevoli, capaci di sviluppare pensiero critico e possibilmente creativi nella risoluzione di problemi e nell'avanzamento di proposte operative. La maggior parte delle persone non diventerà un esperto di informatica o un programmatore, ma indipendentemente dal lavoro che farà potrà sfruttare l'abilità di pensare in modo creativo, di pensare schematicamente laddove necessario e di lavorare sapendo collaborare con altri. Con le parole di Resnick (2018, p. 40): "quando i bambini imparano a usare le tecnologie digitali per esprimersi e condividono le loro idee attraverso il coding, iniziano a vedere se stessi in modo nuovo. Cominciano a vedere la possibilità di contribuire attivamente alla società. Cominciano a vedersi parte del futuro". Una piena cittadinanza digitale sarà raggiunta unicamente se i futuri cittadini impareranno non soltanto a leggere e decifrare le nuove tecnologie, ma anche a crearle e governarle. Ciò che deve essere al centro di percorsi didattici costruiti con questo obiettivo, come fu a suo tempo per la tipografia scolastica (FREINET, 1969), non è la tecnologia ma la comprensione profonda e la concettualizzazione di quel che si consuma e si produce attraverso le infrastrutture del sapere. 
Questa comprensione è difficile da raggiungere in assenza di una pratica attiva di decostruzione e creazione, di percorsi concreti di apertura, sperimentazione e condivisione. Per la scuola e per l'università questo comporta l'avere a che fare con le tecnologie digitali e di rete in modo non passivo, significa accogliere le sfide poste da un ecosistema del sapere estremamente più complesso di quelli che lo hanno preceduto (WEINBERGER, 2012), significa lavorare per consentire agli studenti e ai futuri cittadini di avere un rapporto più maturo e consapevole con i media (GREENHOW; SONNEVEND; AGUR, 2016), le reti e la conoscenza che lì abita (NUSSBAUM-BEACH; HAL, 2011).

Oggi la conoscenza e il sapere si reggono sempre più sulle infrastrutture tecnologiche e sui sistemi che danno loro forma, ed è quindi fondamentale avere esperienza dei fenomeni legati alle reti e riuscire a concettualizzarli in modo non banale. Sta diventando imprescindibile riuscire a comprendere il funzionamento degli algoritmi che reggono le nostre interazioni sociali, i nostri consumi culturali, i nostri acquisti e il nostro rapporto con il sapere, la politica, l'advertising; è essenziale comprenderne le logiche e le ideologie di chi li sviluppa, per essere meno indifesi e più capaci di risposte in grado di incidere sulla realtà (PIREDDU, 2017b). Occorre studiare le logiche culturali dei database e il funzionamento dei metadati, sempre più indispensabili per l'archiviazione, la gestione e la condivisione di risorse e contenuti di ogni tipo. Non si tratta di mere questioni tecniche, ma di dimensioni culturali strategiche e su cui si stanno edificando il presente e il futuro prossimo. Gli algoritmi e i database più diffusi rispondono infatti a chi li crea, nascono con scopi e obiettivi precisi, e in funzione di quelli vengono utilizzati. Non di rado incorporano pregiudizi e bias di chi li ha costruiti e persino degli utilizzatori, e il discorso pubblico su questi temi, così come quello scolastico e troppo spesso anche quello accademico, è ancora caratterizzato da imprecisione quando non da scarsa conoscenza dell'argomento.

Da più parti viene rilevata la diffusione di software in grado di imparare autonomamente, ed è lecito chiedersi quanti educatori e insegnanti possiedono strumenti che consentono loro di comprendere e concettualizzare adeguatamente le sfide poste dal machine learning. O ancora, quanti riescono a riflettere sulle implicazioni profonde di queste innovazioni per la società, l'economia, la cultura o la giustizia sociale. Se la gestione accorta del cambiamento e il governo responsabile delle trasformazioni possono esistere unicamente in presenza di cittadini e classi dirigenti informate, occorre riconoscere che la scuola e l'università non possono permettersi tempi troppo lunghi per un cambiamento efficace e in grado di dare 
risposte alle sfide del presente e del prossimo futuro. Se i sistemi educativi non saranno in grado di lavorare sugli strumenti per la comprensione e la gestione della complessità attuale - non quella del passato - non si potrà sperare di riuscire a governare il cambiamento in senso positivo per la maggior parte dei cittadini. Un cambiamento di questa portata è gestibile unicamente con più studio, più formazione, maggiore consapevolezza e maggiore dimestichezza con le odierne infrastrutture della conoscenza e con le loro logiche di fondo. È possibile chiedere tutele specifiche e norme più efficaci solo se si ha una comprensione almeno di base dei temi in oggetto: cittadini disinformati non potranno che esprimere classi dirigenti poco preparate e scarsamente competenti.

Non si tratta, come sostengono alcuni, di apprendere di più o di meno rispetto a una volta, ma di come e cosa apprendere. Se si continua a pensare che la comprensione di un qualsiasi fenomeno possa essere ancora legata al solo studio di testi, il rischio è quello di far vivere gli studenti in due mondi separati, quello tipografico della scuola e quello reticolare delle tecnologie di rete, limitando ogni dialogo. È opportuno invece lavorare sul tenere insieme la pluralità di dimensioni che caratterizza la complessità delle attuali infrastrutture della conoscenza: leggere e collegare, andare in profondità e comprendere, saper immergersi e dialogare, vedere e interagire, ascoltare e operare, fruire e creare, riflettere e condividere.

Molta parte dell'apprendimento, come si è detto, è legata alla produzione e non unicamente alla fruizione, all'essere soggetti attivi e non soltanto ripetitori di informazioni. Le dimensioni dell'astratto e del concreto, la didattica operativa e la didattica riflessiva hanno bisogno di convivere: porre al centro dei percorsi educativi gli artefatti cognitivi di ogni tipo rende l'apprendimento un processo che avviene concretamente attraverso il ruolo attivo di chi impara. Unicamente in questo modo è possibile per i soggetti coinvolti esplorare il proprio modo di pensare, e comprendere che riflettere sul come si pensa può essere fondamentale per la propria formazione umana e professionale. Le infrastrutture della conoscenza e la fluidità nel loro utilizzo possono aiutare verso questa produzione di domande, di ricerca e riconoscimento di credenze implicite e tendenze nascoste, verso percorsi più consapevoli di riappropriazione dei mezzi di produzione di sapere. 


\section{Riferimenti}

CARRETERO GOMEZ, Stephanie; VUORIKARI, Riina; PUNIE, Yves. DigComp 2.1: The Digital Competence Framework for Citizens with eight proficiency levels and examples of use. Publications Office of the European Union, 2017. Indirizzo Internet: <http://bit.ly/2q9W3gr>. Accesso: 15.09.2017.

FLORIDI, Luciano. La quarta rivoluzione. Come l'infosfera sta trasformando il mondo. Milano: Raffaello Cortina Editore, 2017.

FREINET, C. Le mie tecniche (1967). Firenze: La Nuova Italia, 1969.

GEE JAMES, Paul. The Anti-Education Era. Creating Smarter Students Through Digital Learning. New York: Palgrave Macmillan, 2013.

GREENHOW, Christine; SONNEVEND, Julia; AGUR, Colin. Education and social media: toward a digital future. Cambridge, MA: The MIT Press, 2016.

JOINT RESEARCH CENTRE. European Commission. Digital Competence Framework for Educators (DigCompEdu). 2017. Indirizzo Internet: <http://bit.ly/2micSlG>. Accesso: 15.09.2017.

LANKSHEAR, Colin; KNOBEL, Michele. A cura di, Digital Literacies. New Literacies and Digital Epistemologies. New York: Peter Lang Publishing, 2008.

LIVINGSTONE, Sonia. The Class: Living and Learning in the Digital Age. New York: NYU Press, 2016.

MANOVICH, Lev. Software Culture. Milano: Olivares, 2010.

MARAGLIANO, Roberto; PIREDDU, Mario. Storia e pedagogia nei media. Roma: \#graffi, 2012.

MCLUHAN, Marshall. Gli strumenti del comunicare. Milano: Il Saggiatore, 1997.

MERLEAU-PONTY, Maurice. Fenomenologia della percezione. Milano: Il Saggiatore, 1965.

MORIGGI, Stefano. Connessi. Beati quelli che sapranno pensare con le machine. Milano: San Paolo Edizioni, 2014.

NUSSBAUM-BEACH, Sheryl; HAL, Lani Ritter. The Connected Educator: Learning and Leading in a Digital Age. Bloomington, IN: Solution Tree, 2011.

ONG, Walter Jackson. Oralità e scrittura. Bologna: il Mulino, 1986.

PAPERT, Seymour. I bambini e il computer. Milano: Rizzoli, 1994.

PIREDDU, Mario. Scuola digitale o scuola viva? La logica culturale del Piano Nazionale Scuola Digitale tra mediologia e media education. Media Education. Studi, ricerche, buone pratiche, v. 8, n. 2, p. 160-174, 2017a.

$2017 \mathrm{~b}$.

Algoritmi. Il software culturale che regge le nostre vite. Milano: Luca Sossella Editore,

RESNICK, Mitchel. Come $i$ bambini. Immagina, crea, gioca e condividi. Coltivare la creatività con il Lifelong Kindergarten del MIT. Trento: Erickson, 2018. 
RONCAGLIA, Gino. L'età della frammentazione. Cultura del libro e scuola digitale. Roma-Bari: Laterza, 2018.

WEINBERGER, David. La stanza intelligente. La conoscenza come proprietà della rete. Torino: Codice Edizioni, 2012.

WOLF, Maryanne. Lettore, vieni a casa. Il cervello che legge in un mondo digitale. Milano: Vita e Pensiero, 2018.

Proust e il calamaro. Storia e scienza del cervello che legge. Milano: Vita e Pensiero, 2012. 